\title{
River Flow Estimation from Upstream Flow Records Using Support Vector Machines
}

\author{
Halil Karahan, ${ }^{1}$ Serdar Iplikci, ${ }^{2}$ Mutlu Yasar, ${ }^{1}$ and Gurhan Gurarslan ${ }^{1}$ \\ ${ }^{1}$ Department of Civil Engineering, Faculty of Engineering, Pamukkale University, 20070 Denizli, Turkey \\ ${ }^{2}$ Department of Electrical and Electronics Engineering, Faculty of Engineering, Pamukkale University, 20070 Denizli, Turkey \\ Correspondence should be addressed to Halil Karahan; hkarahan@pau.edu.tr
}

Received 28 January 2014; Revised 22 May 2014; Accepted 6 June 2014; Published 30 June 2014

Academic Editor: Guohe Huang

Copyright @ 2014 Halil Karahan et al. This is an open access article distributed under the Creative Commons Attribution License, which permits unrestricted use, distribution, and reproduction in any medium, provided the original work is properly cited.

\begin{abstract}
A novel architecture for flood routing model has been proposed and its efficiency is validated on several problems by employing support vector machines. The architecture is designed by including the inputs and observed and calculated outflows from the previous time step output. Whole observed data have been used for determining the model parameters in the heuristic methods given in the literature, which constitutes the major disadvantage of the existing approaches. Moreover, using the whole data for training may lead to overtraining problem that causes overfitting of estimations and data. Therefore, in this study, 60-90\% of the data are randomly selected for training and then the remaining data are used for validation. In order to take the effects of the measurement errors into consideration, the data are corrupted by some additive noise. The results show that the proposed architecture improves the model performance under noisy and missing data conditions and that support vector machines can be powerful alternative in flood routing modeling.
\end{abstract}

\section{Introduction}

Flood routing is important in the design of flood protection measures in order to estimate how the proposed measures will affect the behavior of flood waves in rivers so that adequate protection and economic solutions can be found [1]. Flood routing models may be classified as either hydrologic or hydraulic. The hydraulic models solve the Saint-Venant equations by using a numerical method such as finite difference or finite element methods. A great deal of studies based on hydraulic models was developed by various researchers for flood routing [2-8]. These models require the measurement of flow depth and discharges. If detailed topographical surveys of channel cross-sections and roughness at close intervals are not available, hydraulic models are not suitable to serve the purpose of flood routing. In this case, hydrologic models may be used because they can cope with sparse spatial data [9].

Hydrologic models are based on the storage continuity equation and another equation which usually expresses the storage volume as a linear or nonlinear function of inflow and outflow discharges. The Muskingum method is the most widely used hydrologic flood routing method owing to its simplicity [10]. Many researchers made studies on the parameter estimation of Muskingum flood routing models [11-14]. Performances of the Muskingum models depend on the selection of the appropriate storage equation and the optimal parameter estimation of these models. Even if the parameters of storage equation are determined as optimum, every flood event may not be adequately represented. In particular, this problem occurs in a flood event containing more than one peak and/or having substantially lateral flow.

In order to overcome this problem, data-driven flood routing models based on support vector machines (SVM) need to be developed. SVM is based on statistical learning theory and structural risk minimization principle and can solve any regression problems without getting stuck into local minima. They achieve the global solution by transforming the regression problem into a quadratic programming $(\mathrm{QP})$ problem and then solving it by a QP solver. Finding global solution and possessing higher generalization capability constitute the major advantages of the SVM algorithms over other regression techniques [15]. In the last decade, SVMbased algorithms have been developed very rapidly and have 


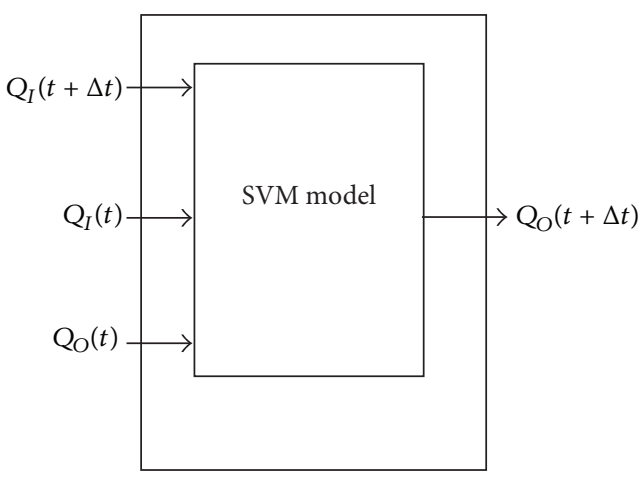

(a)



(b)

FIgURE 1: (a) Training phase and (b) prediction phase.

been applied to many areas $[16,17]$. In particular, the SVM have been used for modeling and prediction purposes for solving some problems in the hydrology area of research and application [18-29].

The fact that the whole observed data has been used for determining the model parameters in the abovementioned heuristic methods constitutes the major disadvantage of these approaches. This may lead to overtraining problem that degrades generalization capability [34]. In order to prevent overtraining, some part of the data is used for training and the remaining part is spared for validation. Thus, in this study, 60-90\% of the data are randomly selected for training (for determining model parameters) and then the remaining unseen data are used for validation. Therefore, in this study, novel model architecture has been proposed and its efficiency is validated on several problems. This is organized as follows.

In the next section, first, the proposed training and prediction procedures are introduced and then the training algorithm is explained in detail for SVM in the following subsection. In Section 3, the three numerical applications are investigated to show the efficiency of the proposed method by comparing SVM to other methods when all data are used for training and also to each other when only some portion of the data is used for training. The simulation results are discussed in Section 4.

\section{Model Development}

In this study, we have employed SVM in flood routing modeling for prediction of its future behavior. For this purpose, as can be seen in Figure 1(a), an SVM model of the flood routing is obtained in the training phase by using a training set $T$ as given by

$$
\begin{gathered}
T=\left\{Q_{I}(t+k \Delta t), Q_{I}(t+(k-1) \Delta t), Q_{O}(t+(k-1) \Delta t)\right. \\
\left.; Q_{O}(t+k \Delta t)\right\}_{k=1}^{k=N},
\end{gathered}
$$

where $Q_{I}(t)$ and $Q_{O}(t)$ are the measured input and output flow rates at time $t$, respectively, $\Delta t$ is the time interval between the successive measurements, and $N$ is the number of training data. For sake of the simplicity, the data set $T$ can be represented more compactly as $T=\left\{\mathbf{x}_{k} ; y(k)\right\}_{k=1}^{k=N}$, where $\mathbf{x}_{k} \in X \subseteq \mathfrak{R}^{3}$ is the $k$ th input data point in the input space and $y(k) \in Y \subseteq \mathfrak{R}$ is the corresponding output value; that is,

$$
\begin{gathered}
\mathbf{x}_{k}\left[Q_{I}(t+k \Delta t), Q_{I}(t+(k-1) \Delta t), Q_{O}(t+(k-1) \Delta t)\right]^{T} ; \\
y(k)=Q_{O}(t+k \Delta t) .
\end{gathered}
$$

In the modeling phase, to obtain a model that represents the relationship between the input and output data points is desired. The training data set $T$ is to be used to obtain an approximate model of the flood. Once the SVM model of the flood routing is obtained, then its future behavior can be predicted by the mechanism depicted in Figure 1(b), where the predicted output of the model is delayed by $\Delta t$ and then fed back to the model itself as the third input thereby making the model more realistic to predict the flood.

In this section, the $\varepsilon$-SVR algorithm, SVM regression algorithm used in this study, is described briefly. The primal form of an SVM regression model is given by (3), which is linear in a higher dimensional feature space $\boldsymbol{F}$.

Consider

$$
\widehat{y}\left(\mathbf{x}_{i}\right)=\left\langle\mathbf{w}, \boldsymbol{\Phi}\left(\mathbf{x}_{i}\right)\right\rangle+b,
$$

where $\mathbf{w}$ is a vector in the feature space $\boldsymbol{F}, \boldsymbol{\Phi}(\cdot)$ is a mapping from the input space to the feature space, $b$ is the bias term, and $\langle\cdot\rangle$ is the inner product operation in the feature space. The SVM regression algorithm looks upon the regression problem as an optimization problem in dual space, where the model is given by

$$
\widehat{y}\left(\mathbf{x}_{i}\right)=\sum_{j=1}^{N} \alpha_{j} K\left(\mathbf{x}_{i}, \mathbf{x}_{j}\right)+b,
$$

where $\alpha_{j}$ 's are the coefficients of each training data point and $K\left(\mathbf{x}_{i}, \mathbf{x}_{j}\right)$ is a kernel function. The kernel function handles the inner product in the feature space; that is, $K\left(\mathbf{x}_{i}, \mathbf{x}_{j}\right)=$ $\left\langle\boldsymbol{\Phi}\left(\mathbf{x}_{i}\right), \boldsymbol{\Phi}\left(\mathbf{x}_{j}\right)\right\rangle$, and hence the explicit form of $\boldsymbol{\Phi}(\mathbf{x})$ does not need to be known. In this study, we have used the radial basis kernel function given by

$$
K\left(\mathbf{x}_{i}, \mathbf{x}_{j}\right)=K_{i j}=\exp \left(-\frac{\left\|\mathbf{x}_{i}-\mathbf{x}_{j}\right\|^{2}}{2 \sigma^{2}}\right),
$$


TABLE 1: Comparison of the observed and computed outflows for Wilson data.

\begin{tabular}{|c|c|c|c|c|c|c|}
\hline Time (h) & Input (cms) & Output (cms) & $\begin{array}{c}\text { NLMM-L } \\
{[30]}\end{array}$ & $\begin{array}{c}\text { VPWFDM-L } \\
{[31]}\end{array}$ & $\begin{array}{l}\text { FIS } \\
{[32]}\end{array}$ & SVM \\
\hline 0 & 22 & 22 & 22 & 22 & 22.0 & 22.00 \\
\hline 6 & 23 & 21 & 21.71 & 20.66 & 20.9 & 20.93 \\
\hline 12 & 35 & 21 & 22.02 & 21.5 & 21.0 & 20.98 \\
\hline 18 & 71 & 26 & 26.08 & 25.79 & 26.0 & 25.92 \\
\hline 24 & 103 & 34 & 33.51 & 33.71 & 34.0 & 34.04 \\
\hline 30 & 111 & 44 & 42.83 & 44.65 & 44.0 & 43.97 \\
\hline 36 & 109 & 55 & 55.44 & 54.37 & 57.1 & 55.04 \\
\hline 42 & 100 & 66 & 66.67 & 65.72 & 66.2 & 65.96 \\
\hline 48 & 86 & 75 & 75.77 & 75.78 & 75.0 & 75.06 \\
\hline 54 & 71 & 82 & 82.12 & 82.55 & 82.0 & 81.93 \\
\hline 60 & 59 & 85 & 84.78 & 84.65 & 85.4 & 84.95 \\
\hline 66 & 47 & 84 & 83.42 & 84.07 & 84.0 & 84.08 \\
\hline 72 & 39 & 80 & 79.44 & 79.36 & 80.0 & 79.93 \\
\hline 78 & 32 & 73 & 72.48 & 72.67 & 73.0 & 72.92 \\
\hline 84 & 28 & 64 & 64.08 & 63.75 & 64.0 & 64.02 \\
\hline 90 & 24 & 54 & 54.58 & 54.53 & 54.0 & 54.04 \\
\hline 96 & 22 & 44 & 45.22 & 44.87 & 44.0 & 43.96 \\
\hline 102 & 21 & 36 & 36.34 & 36.24 & 35.9 & 36.04 \\
\hline 108 & 20 & 30 & 29.21 & 29.5 & 29.9 & 29.96 \\
\hline 114 & 19 & 25 & 24.21 & 24.56 & 25.3 & 25.04 \\
\hline 120 & 19 & 22 & 20.96 & 21.31 & 21.7 & 21.96 \\
\hline 126 & 18 & 19 & 19.41 & 19.39 & 19.1 & 19.04 \\
\hline SSE & & & 9.823 & 5.178 & 4.830 & 0.056 \\
\hline
\end{tabular}

where $\|\cdot\|$ is the Euclidean norm and $\sigma$ is the width parameter. In the model (4), a training point $\mathbf{x}_{j}$ corresponding to a nonzero $\alpha_{j}$ value is referred to as the support vector. The $\varepsilon$ SVR algorithm employs Vapnik's $\varepsilon$-insensitive loss function $L(\varepsilon, y, \widehat{y})$ given by

$$
L(\varepsilon, y, \widehat{y})= \begin{cases}0 & y-\hat{y} \leq \varepsilon \\ y-\widehat{y} & y-\hat{y}>\varepsilon\end{cases}
$$

and formulates the primal form of the regression problem as follows:

$$
\min _{\mathbf{w}, b, \xi, \xi^{*}} P_{\varepsilon}=\frac{1}{2}\|\mathbf{w}\|^{2}+C \sum_{i=1}^{N}\left(\xi_{i}+\xi_{i}^{*}\right)
$$

subject to the constraints

$$
\begin{gathered}
y\left(\mathbf{x}_{i}\right)-\left\langle\mathbf{w}, \boldsymbol{\Phi}\left(\mathbf{x}_{i}\right)\right\rangle-b \leq \varepsilon+\xi_{i}, \quad i=1, \ldots, N \\
\left\langle\mathbf{w}, \boldsymbol{\Phi}\left(\mathbf{x}_{i}\right)\right\rangle+b-y\left(\mathbf{x}_{i}\right) \leq \varepsilon+\xi_{i}^{*}, \quad i=1, \ldots, N \\
\xi_{i}, \xi_{i}^{*} \geq 0, \quad i=1, \ldots, N
\end{gathered}
$$

where $\xi_{i}$ 's and $\xi_{i}^{*}$ 's are slack variables, $\varepsilon$ is the upper value of tolerable error for the output, and $C$ is a regularization parameter that provides a compromise between the model complexity and the degree of tolerance to the errors larger than $\varepsilon$. Dual form of the optimization problem becomes a quadratic programming $(\mathrm{QP})$ problem as follows:

$$
\begin{aligned}
\min _{\beta, \beta^{*}} D_{\varepsilon}= & \frac{1}{2} \sum_{i=1}^{N} \sum_{j=1}^{N} K_{i j}\left(\beta_{i}-\beta_{i}^{*}\right)\left(\beta_{j}-\beta_{j}^{*}\right)+\varepsilon \sum_{i=1}^{N}\left(\beta_{i}+\beta_{i}^{*}\right) \\
& -\sum_{i=1}^{N} y\left(\mathbf{x}_{i}\right)\left(\beta_{i}-\beta_{i}^{*}\right),
\end{aligned}
$$

subject to the constraints

$$
0 \leq \beta_{i}, \beta_{i}^{*} \leq C, \quad \sum_{i=1}^{N}\left(\beta_{i}-\beta_{i}^{*}\right)=0, \quad i=1, \ldots, N .
$$

Solution of the QP problem gives the optimum values of $\beta_{i}$ 's and $\beta_{i}^{*}$ 's. The value of $b$ in the model is determined as follows: the condition $y\left(\mathbf{x}_{i}\right)-\hat{y}\left(\mathbf{x}_{i}\right)=\varepsilon$ is satisfied for each support vector $\mathbf{x}_{i}$ for which the condition $0 \leq \beta_{i}-\beta_{i}^{*} \leq C$ holds. If $\alpha_{j}$ is defined to be the new coefficient of $\mathbf{x}_{j}$ for $j=1, \ldots, N$ as $\alpha_{j}=\beta_{j}-\beta_{j}^{*}$, then we obtain an SVM model as given by (4). Furthermore, if the support vectors are considered only, then the model becomes

$$
\widehat{y}\left(\mathbf{x}_{i}\right)=\sum_{\substack{j=1 \\ j \in S \mathrm{~V}}}^{\# \mathrm{SV}} \alpha_{j} K\left(\mathbf{x}_{i}, \mathbf{x}_{j}\right)+b,
$$


TABLE 2: Comparison of the observed and routed outflows for Viessman and Lewis data.

\begin{tabular}{|c|c|c|c|c|c|}
\hline Time $(\mathrm{h})$ & Input (cms) & Output (cms) & $\begin{array}{c}\text { NLMM-L } \\
{[30]}\end{array}$ & $\begin{array}{c}\text { VPWFDM-L } \\
\text { [31] }\end{array}$ & SVM \\
\hline 0 & 166.2 & 118.4 & 166.2 & 118.40 & 118.40 \\
\hline 1 & 263.6 & 197.4 & 166.2 & 182.23 & 198.71 \\
\hline 2 & 365.3 & 214.1 & 263.25 & 262.43 & 215.39 \\
\hline 3 & 580.5 & 402.1 & 346.87 & 362.43 & 403.06 \\
\hline 4 & 594.7 & 518.2 & 505.25 & 496.97 & 519.25 \\
\hline 5 & 662.6 & 523.9 & 563.12 & 559.30 & 526.01 \\
\hline 6 & 920.3 & 603.1 & 620.77 & 633.91 & 604.67 \\
\hline 7 & 1568.8 & 829.7 & 773.8 & 803.13 & 831.03 \\
\hline 8 & 1775.5 & 1124.2 & 1109.49 & 1150.33 & 1122.98 \\
\hline 9 & 1489.5 & 1379 & 1381.64 & 1417.85 & 1377.61 \\
\hline 10 & 1223.3 & 1509.3 & 1460.43 & 1433.22 & 1508.11 \\
\hline 11 & 713.6 & 1379 & 1389.1 & 1345.15 & 1378.08 \\
\hline 12 & 645.6 & 1050.6 & 1133.57 & 1115.25 & 1049.46 \\
\hline 13 & 1166.7 & 1013.7 & 890.74 & 968.99 & 1012.38 \\
\hline 14 & 1427.2 & 1013.7 & 982.97 & 994.50 & 1012.35 \\
\hline 15 & 1282.8 & 1013.7 & 1167.97 & 1022.09 & 1014.47 \\
\hline 16 & 1098.7 & 1209.1 & 1236.21 & 1216.84 & 1207.94 \\
\hline 17 & 764.6 & 1248.8 & 1192.89 & 1231.67 & 1246.96 \\
\hline 18 & 458.7 & 1002.4 & 1019.78 & 1023.80 & 1000.48 \\
\hline 19 & 351.1 & 713.6 & 743.04 & 689.20 & 714.04 \\
\hline 20 & 288.8 & 464.4 & 501.27 & 473.12 & 466.09 \\
\hline 21 & 228.8 & 325.6 & 345.06 & 351.18 & 327.39 \\
\hline 22 & 170.2 & 265.6 & 245.18 & 266.89 & 266.12 \\
\hline \multirow[t]{2}{*}{23} & 143 & 222.6 & 168.87 & 194.24 & 224.38 \\
\hline & & & 73399.33 & 26185 & 43.37 \\
\hline
\end{tabular}

where \#SV stands for the number of support vectors in the model $[19,35]$ The SVM model is sparse in the sense that the whole training data are represented by only support vectors. The parameters of $\varepsilon$-SVR are the maximum tolerable error $\varepsilon$ at the output, the regularization parameter $C$, the number of training patterns $N$, and the width parameter $\sigma$. The major advantage of the $\varepsilon$-SVR algorithm is that it allows for the determination of the maximum total training error beforehand by choosing a proper $\varepsilon$ value.

\section{Numerical Applications}

In this study, we have tested modeling and prediction performance of the proposed SVM structure on three different flood problems. For each problem, we have gathered some artificial and real-world data for modeling purposes. In this comparative work, we have split our comparisons into two cases. In Case I, in order to have a basis for fair comparisons to other methods, all of the gathered data are used for only training of SVM structure. In Case II, only some portions, $\mu$, of the data are used for training, while remaining data are spared for validation and then the SVM approach is compared to other models given in the literature. For both cases in the training phase, all variables in each data set are normalized to the interval $[0,1]$ and then an appropriate data set for training is formed. Afterwards, SVM model is obtained to give least possible training plus validation errors.

3.1. Application to Wilson Data [36]. Data sets reported by Wilson are known to present a nonlinear relationship between weighted discharge and storage and used extensively in the literature as a benchmark problem. The number of data in this example is 22. The comparison of the SVM to other methods with respect to the prediction performances for Example 1 is given in Table 1.

The Wilson flood data were modeled by Karahan et al. (2014) using a nonlinear Muskingum model incorporating lateral flow (NLMM-L) and SSE value was found as 9.823. Chu (2009) presented the combined application of fuzzy inference system (FIS) and Muskingum model in flood routing. Chu (2009) found the SSE value as 4.830. More recently, Karahan et al. (2014) have proposed a variable-parameter nonlinear Muskingum model incorporating lateral flow with a weighted finite difference method [VPWFDM-L] and applied this model to Wilson data. Karahan et al. (2014) have reported the SSE value as 5.178. When the SVM model is employed for the same flood data, the SSE value has been found as 0.056 , which is much better than that of other methods. 
TABLE 3: Comparison of the observed and routed outflows for the River Wyre data.

\begin{tabular}{|c|c|c|c|c|c|}
\hline Time (h) & Input (cms) & Output (cms) & $\begin{array}{c}\text { LMM-L } \\
\text { [33] }\end{array}$ & $\begin{array}{c}\text { NLMM-L } \\
{[30]}\end{array}$ & SVM \\
\hline 0 & 2.6 & 8.3 & 8.3 & 8.3 & 8.300 \\
\hline 1 & 4.2 & 9 & 8.2 & 8.51 & 9.092 \\
\hline 2 & 12.3 & 9.9 & 8.1 & 8.79 & 10.020 \\
\hline 3 & 25.4 & 10.2 & 12.7 & 10.94 & 10.338 \\
\hline 4 & 24.1 & 18.9 & 27.9 & 20.28 & 19.063 \\
\hline 5 & 20.3 & 35.9 & 39.9 & 37.54 & 35.980 \\
\hline 6 & 23.3 & 51.8 & 45.7 & 49.07 & 51.815 \\
\hline 7 & 27.7 & 59.4 & 52.2 & 55.11 & 59.327 \\
\hline 8 & 27.7 & 63.3 & 61.4 & 62.5 & 63.279 \\
\hline 9 & 26.9 & 69.6 & 68.9 & 71.44 & 69.482 \\
\hline 10 & 24.8 & 76.7 & 74.7 & 78.03 & 76.649 \\
\hline 11 & 26.9 & 82 & 77.2 & 82.07 & 82.051 \\
\hline 12 & 33.7 & 85.3 & 79.8 & 83.72 & 85.234 \\
\hline 13 & 33.9 & 89 & 87.8 & 87.43 & 88.901 \\
\hline 14 & 27.8 & 94.6 & 95.5 & 95.49 & 94.514 \\
\hline 15 & 20.8 & 98.8 & 97.7 & 100.88 & 98.683 \\
\hline 16 & 15.6 & 98 & 94.4 & 99.29 & 97.902 \\
\hline 17 & 11.9 & 91.8 & 87.9 & 92.06 & 91.728 \\
\hline 18 & 9.5 & 82.3 & 79.8 & 82.22 & 82.217 \\
\hline 19 & 7.8 & 72 & 71.5 & 71.75 & 72.086 \\
\hline 20 & 6.5 & 61.9 & 63.6 & 61.91 & 61.824 \\
\hline 21 & 5.8 & 53 & 56.1 & 53.12 & 53.068 \\
\hline 22 & 5 & 45.6 & 49.6 & 45.47 & 45.560 \\
\hline 23 & 4.8 & 39.2 & 43.7 & 39.14 & 39.259 \\
\hline 24 & 4.5 & 33.8 & 38.8 & 33.76 & 33.764 \\
\hline 25 & 4.1 & 29.3 & 34.6 & 29.55 & 29.363 \\
\hline 26 & 3.7 & 26.2 & 30.9 & 26.12 & 26.085 \\
\hline 27 & 3.4 & 23.5 & 27.7 & 23.2 & 23.496 \\
\hline 28 & 3.2 & 21.2 & 24.8 & 20.67 & 21.289 \\
\hline 29 & 2.9 & 19.2 & 22.3 & 18.52 & 19.367 \\
\hline 30 & 2.8 & 17.7 & 20.1 & 16.71 & 17.747 \\
\hline 31 & 2.6 & 16.4 & 18.2 & 15.12 & 16.527 \\
\hline SSE & & & 468.840 & 53.708 & 0.253 \\
\hline
\end{tabular}

3.2. Application to Viessman and Lewis Data [37]. This example is based on inflow and outflow hydrographs exhibiting linear characteristics and presents a relatively difficult prediction problem for flood routing, where there exist two successively active floods. The number of data in this example is 24 .

Table 2 shows the comparison results numerically. It is obviously seen that the SVM method outperforms others in prediction of the flood dynamics, which can be attributed to the proposed training and prediction structures and also the generalization potentials of SVM approach.

As can be seen from Table 2, the SSE values are obtained as 73399.33 for the NLMM-L model, 26185 for the VPWFDM$\mathrm{L}$ model, and 43.37 for the SVM model, respectively. It is observed from the results of Table 3 that the SSE value $(0.253)$ obtained by the SVM model for the River Wyre data is better than those obtained when the LMM-L and NLMM-L models are used.
3.3. Application to River Wyre [33]. For the River Wyre data, the flood volume between the inflow and the outflow sections is $25 \mathrm{~km}$, along which there are lateral flows that considerably contribute to the flood [33]. Moreover, the input hydrograph has multiple peaks. The number of data in this example is 32 .

In the literature, the River Wyre flood data were first modeled by O'Donnell (1985) using a linear Muskingum model incorporating lateral flow (LMM-L) and the SSE value was found as 468.840. Recently, Karahan et al. (2014) have applied NLMM-L model to River Wyre flood data and have reported the SSE value as 53.708. It is observed from the results that the SSE value (0.253) obtained by the SVM model for the River Wyre data is better than those obtained when the LMM-L and NLMM-L models are used.

3.4. Verification of Model Robustness. In Sections 3.1-3.3, the SVM models have been obtained by using whole data and then compared to other methods in the literature. The 
TABLE 4: Average SSE values of the SVM approach for Wilson data, Viessman and Lewis data, and River Wyre data.

\begin{tabular}{|c|c|c|c|c|c|}
\hline Data & $\mu$ & $\delta=0$ & $\delta=0.01$ & $\delta=0.05$ & $\delta=0.10$ \\
\hline \multirow{4}{*}{ Wilson } & 0.9 & 1.389 & 1.627 & 2.013 & 2.691 \\
\hline & 0.8 & 3.486 & 4.120 & 4.160 & 7.738 \\
\hline & 0.7 & 7.284 & 8.473 & 8.835 & 10.818 \\
\hline & 0.6 & 12.540 & 14.872 & 17.878 & 21.755 \\
\hline \multirow{4}{*}{ Viessman and Lewis } & 0.9 & 12460.720 & 14446.716 & 18456.445 & 21172.915 \\
\hline & 0.8 & 34631.312 & 48888.423 & 50163.966 & 58837.181 \\
\hline & 0.7 & 83005.647 & 84387.246 & 112145.221 & 100247.301 \\
\hline & 0.6 & 124339.197 & 124324.518 & 118768.183 & 131235.761 \\
\hline \multirow{4}{*}{ River Wyre } & 0.9 & 5.760 & 9.895 & 11.922 & 7.788 \\
\hline & 0.8 & 218.236 & 55.846 & 70.096 & 289.540 \\
\hline & 0.7 & 291.692 & 161.367 & 133.620 & 363.987 \\
\hline & 0.6 & 331.883 & 271.468 & 239.053 & 440.319 \\
\hline
\end{tabular}

results have shown that there is good agreement between the predicted and measured outflows for the three examples under investigation. However, it is possible that there may be some erroneous and/or missing measurements in practical applications. In order to test the performance of the proposed SVM model under such conditions, input data have been corrupted by additive uniformly distributed noise with zero mean. The noisy data are obtained as $[38,39]$

$$
Q_{I}^{*}(t)=Q_{I}(t)+\lambda \delta Q_{I}(t),
$$

where $Q_{I}(t)$ and $Q_{I}^{*}(t)$ stand for the noiseless and noisy input flow rates at time $t$, respectively, $\lambda$ represents the measurements errors that are distributed uniformly between -1 and 1 , and $\delta$ is a noise level scalar between 0.0 and 0.1 . In this study, various noise level conditions, namely, 0.01, 0.05, and 0.10 , are considered and also it is assumed that some portions (10 to 40 percent) of the data are missing in order to investigate the effects of the missing data on the model performance. In order to get more reliable results, the tests have been performed at least 100 times for each case and then their average SSE values have been given in Table 4 .

As can be seen from Table 4, only $\mu$ portion of data is used for training, while its remaining part is spared for validation. The test data are selected randomly out of the whole data set. It is observed from numerical results that the SVM method provides excellent prediction performance when there is no measurement noise $(\delta=0)$ and the nearly whole data are used $(\mu=0.9)$. On the other hand, as the level of the measurement noise and the portion of the missing data are increased, the model performance decreases expectedly. Still, in the worst case $(\delta=0.1$ and $\mu=0.6)$ the proposed SVM method provides acceptable performance.

\section{Conclusions}

In this study, a novel architecture for flood routing model has been proposed and its efficiency is validated on three different flood routing problems by employing SVM approach. Proposed model is designed including the inputs and observed and calculated outflows from the previous time step output, thereby making the model more realistic. The SVM approach has been implemented to capture the dynamics of the investigated floods from the observed data. In this study, higher generalization capabilities have motivated us to employ the SVM structure. After completing the learning phase, the model has been performed to predict the routing outflows. The proposed model has also been compared to the different models in the literature.

The simulation results have revealed that when combined with the powerful modeling tools, such as SVM, the proposed architecture exhibits excellent modeling and prediction performances for flood routing problems under investigation. The results have also demonstrated that the proposed model provides better prediction performance than the ones existing in the literature when whole data are used for training. Furthermore, SVM approach has been employed when only some portions $(60-90 \%)$ of the data are used for training, and it has been observed that SVM maintains its prediction performance up to an acceptable level even if only $60 \%$ of the data are used for training under noisy condition. Consequently, the proposed model possesses higher applicability potential in forecasting outflows with different inflow patterns and thus it can be employed for solving flood routing problems.

\section{Conflict of Interests}

The authors declare that there is no conflict of interests regarding the publication of this paper.

\section{References}

[1] V. P. Singh and R. C. McCann, "Some notes on Muskingum method of flood routing," Journal of Hydrology, vol. 48, no. 34, pp. 343-361, 1980.

[2] M. Amein, "An implicit method for natural flood routing," Water Resources Research, vol. 4, no. 4, pp. 719-726, 1968.

[3] R. A. Baltzer and C. Lai, "Computer simulation of unsteady flows in waterway," Journal of Hydraulic Division, vol. 94, no. 4, pp. 1083-1117, 1968. 
[4] M. Amein and C. S. Fang, "Implicit flood routing in natural channels," Journal of Hydraulic Research, vol. 96, no. 12, pp. 2481-2500, 1970.

[5] M. Amein and L. H. Chu, "Implicit numerical modeling of unsteady flows," Journal of Hydraulic Division, vol. 101, no. 6, pp. 717-731, 1975.

[6] M. B. Abbott and J. A. Cunge, Engineering Applications of Computational Hydraulics, Marshfield, Pitman, NJ, USA, 1982.

[7] U.S. Army Corps of Engineering (USACE), HEC-RAS River Analysis System Hydraulic Reference Manual (Version 3.1), USACE-HEC, Davis, Calif, USA, 2002.

[8] Danish Hydrological Institute (DHI), User's Manual and Technical References for MIKE 11 (Version 2003b), Danish Hydrological Institute (DHI), Hørsholm, Denmark, 2003.

[9] H. Karahan, G. Gurarslan, and Z. W. Geem, "Parameter estimation of the nonlinear muskingum flood routing model using a hybrid harmony search algorithm," Journal of Hydrologic Engineering, vol. 18, no. 3, pp. 352-360, 2013.

[10] H. Karahan, "Predicting Muskingum flood routing parameters using spreadsheets," Computer Applications in Engineering Education, vol. 20, no. 2, pp. 280-286, 2012.

[11] A. Aldama, "Least-squares parameter estimation for Muskingum flood routing," Journal of Hydraulic Engineering, vol. 116, no. 4, pp. 580-586, 1990.

[12] A. Das, "Parameter estimation for Muskingum models," Journal of Irrigation and Drainage Engineering, vol. 130, no. 2, pp. 140$147,2004$.

[13] S. Mohan, "Parameter estimation of nonlinear Muskingum models using genetic algorithm," Journal of Hydraulic Engineering, vol. 123, no. 2, pp. 137-142, 1997.

[14] J. H. Kim, Z. W. Geem, and E. S. Kim, "Parameter estimation of the nonlinear Muskingum model using harmony search," Journal of the American Water Resources Association, vol. 37, no. 5, pp. 1131-1138, 2001.

[15] V. N. Vapnik, Statistical Learning Theory, Adaptive and Learning Systems for Signal Processing, Communications, and Control, Wiley- Interscience, New York, NY, USA, 1998.

[16] N. Cristianini and J. S. Taylor, An Introduction to Support Vector Machines and Other Kernel-Based Learning Methods, Cambridge University Press, New York, NY, USA, 2000.

[17] B. Schölkopf, C. J. C. Burges, and A. J. Smola, Advances in Kernel Methods: Support Vector Learning, MIT Press, Cambridge, Mass, USA, 1999.

[18] M. Behzad, K. Asghari, M. Eazi, and M. Palhang, "Generalization performance of support vector machines and neural networks in runoff modeling," Expert Systems with Applications, vol. 36, no. 4, pp. 7624-7629, 2009.

[19] X. Zhang, R. Srinivasan, and M. van Liew, "Approximating SWAT model using artificial neural network and support vector machine," Journal of the American Water Resources Association, vol. 45, no. 2, pp. 460-474, 2009.

[20] G. F. Lin, Y. C. Chou, and M. C. Wu, “Typhoon flood forecasting using integrated two-stage support vector machine approach," Journal of Hydrology, vol. 486, pp. 334-342, 2013.

[21] E. K. Kakaei Lafdani, A. M. Moghaddam Nia, and A. Ahmadi, "Daily suspended sediment load prediction using artificial neural networks and support vector machines," Journal of Hydrology, vol. 478, pp. 50-62, 2013.

[22] Z.B. Yu, D. Liu, H.S. Lu, L. Xiang, and Y. H. Zhu, "A multi-layer soil moisture data assimilation using and ensemble particle filter," Journal of Hydrology, vol. 475, pp. 53-64, 2012.
[23] O. Kisi, "Modeling discharge-suspended sediment relationship using least square support vector machine," Journal of Hydrology, vol. 456-457, pp. 110-120, 2012.

[24] O. Kisi and M. Cimen, "A wavelet-support vector machine conjunction model for monthly streamflow forecasting," Journal of Hydrology, vol. 399, no. 1-2, pp. 132-140, 2011.

[25] H. Yoon, S. C. Jun, Y. Hyun, G. O. Bae, and K.K. Lee, "A comparative study of artificial neural networks and support vector machines for predicting groundwater levels in a coastal aquifer," Journal of Hydrology, vol. 396, pp. 128-138, 2011.

[26] S. T. Chen, P. S. Yu, and Y. H. Tang, "Statistical downscaling of daily precipitation using support vector machines and multivariate analysis," Journal of Hydrology, vol. 385, no. 1-4, pp. 1322, 2010.

[27] G.-F. Lin, G.-R. Chen, P.-Y. Huang, and Y.-C. Chou, "Support vector machine-based models for hourly reservoir inflow forecasting during typhoon-warning periods," Journal of Hydrology, vol. 372, no. 1-4, pp. 17-29, 2009.

[28] S. Tripathi, V. V. Srinivas, and R. S. Nanjundiah, "Dowinscaling of precipitation for climate change scenarios: a support vector machine approach," Journal of Hydrology, vol. 330, no. 3-4, pp. 621-640, 2006.

[29] T. Asefa, M. Kemblowski, M. McKee, and A. Khalil, "Multitime scale stream flow predictions: the support vector machines approach," Journal of Hydrology, vol. 318, no. 1-4, pp. 7-16, 2006.

[30] H. Karahan, G. Gurarslan, and Z. W. Geem, "A new nonlinear muskingum flood routing model incorporating lateral flow," Engineering Optimization. In press, 2014.

[31] H. Karahan, "Discussion of "improved nonlinear Muskingum model with variable exponent parameter"', Journal of Hydrologic Engineering, 2014.

[32] H.-J. Chu, "The Muskingum flood routing model using a neurofuzzy approach," KSCE Journal of Civil Engineering, vol. 13, no. 5, pp. 371-376, 2009.

[33] T. O’Donnell, "A direct three-parameter Muskingum procedure incorporating lateral inflow.," Hydrological Sciences Journal, vol. 30, no. 4, pp. 479-496, 1985.

[34] H. R. Maier and G. C. Dandy, "Neural networks for the prediction and forecasting of water resources variables: a review of modelling issues and applications," Environmental Modelling \& Software, vol. 15, no. 1, pp. 101-124, 2000.

[35] A. J. Smola and B. Schölkopf, "A tutorial on support vector regression," NeuroCOLT Tech. Rep. NC-TR-98-030, Royal Holloway College, University of London, 1998.

[36] E. M. Wilson, Engineering Hydrology, MacMillan Education, Hampshire, UK, 1974.

[37] W. Viessman Jr. and G. L. Lewis, Introduction to Hydrology, Pearson Education Inc, Upper Saddle River, NJ, USA, 2003.

[38] H. Karahan and M. T. Ayvaz, "Simultaneous parameter identification of a heterogeneous aquifer system using artificial neural networks," Hydrogeology Journal, vol. 16, no. 5, pp. 817-827, 2008.

[39] G. Gurarslan, Identification of groundwater contaminant source locations and release histories by using differential evolution algorithm [Ph. D. thesis], Department of Civil Engineering, Pamukkale University, Denizli, Turkey, 2011, (Turkish). 




Advances in

Operations Research

mansans

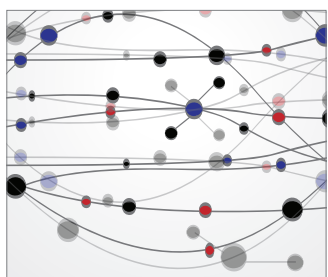

The Scientific World Journal
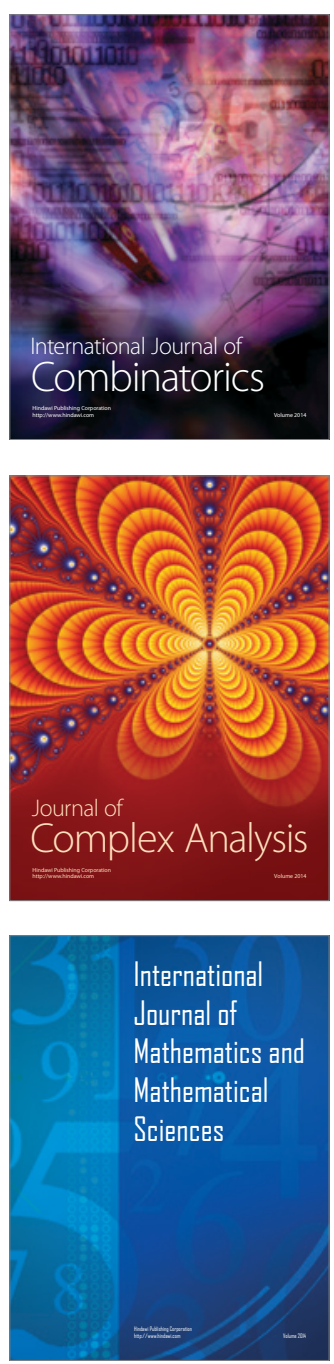
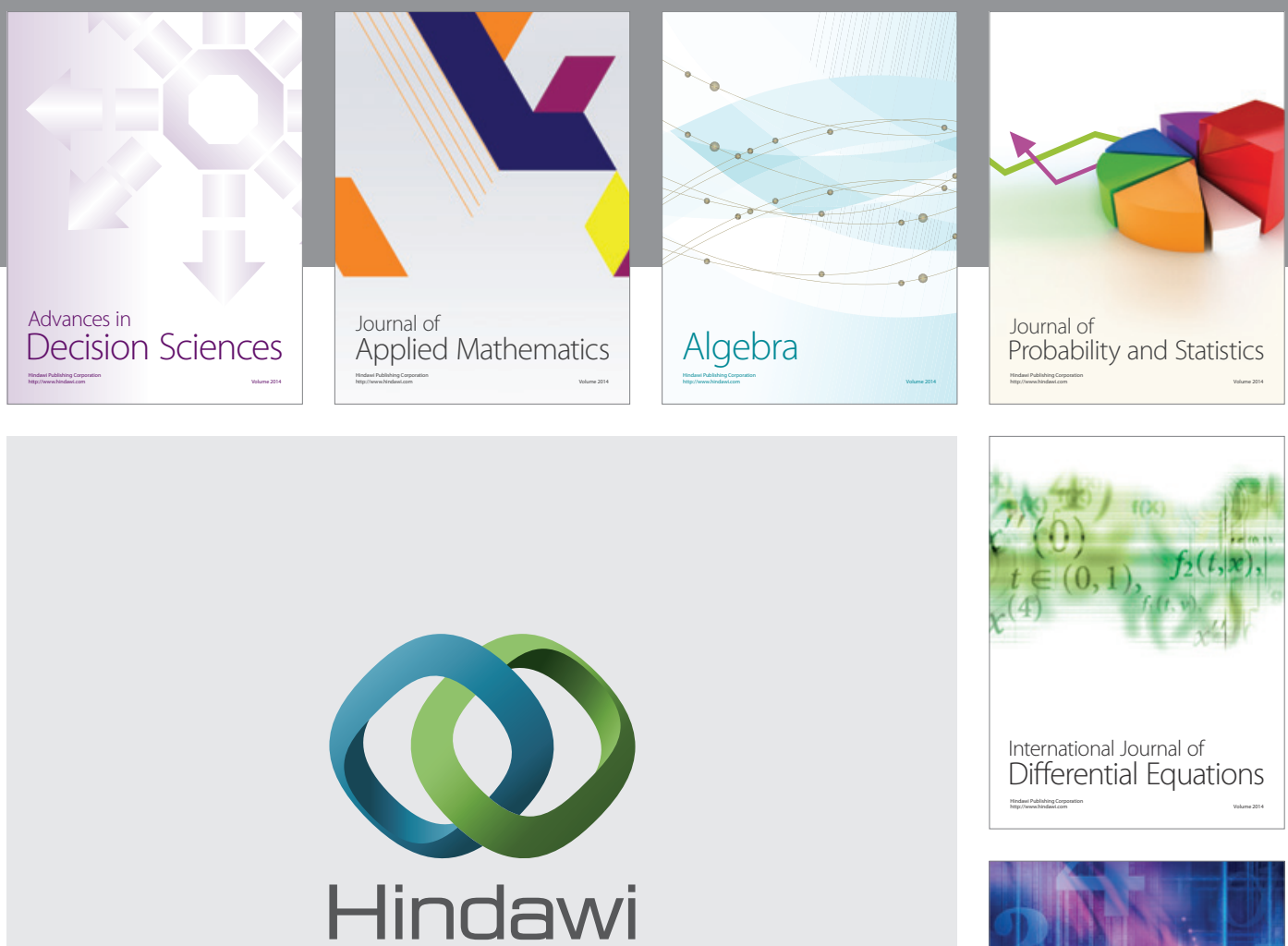

Submit your manuscripts at http://www.hindawi.com
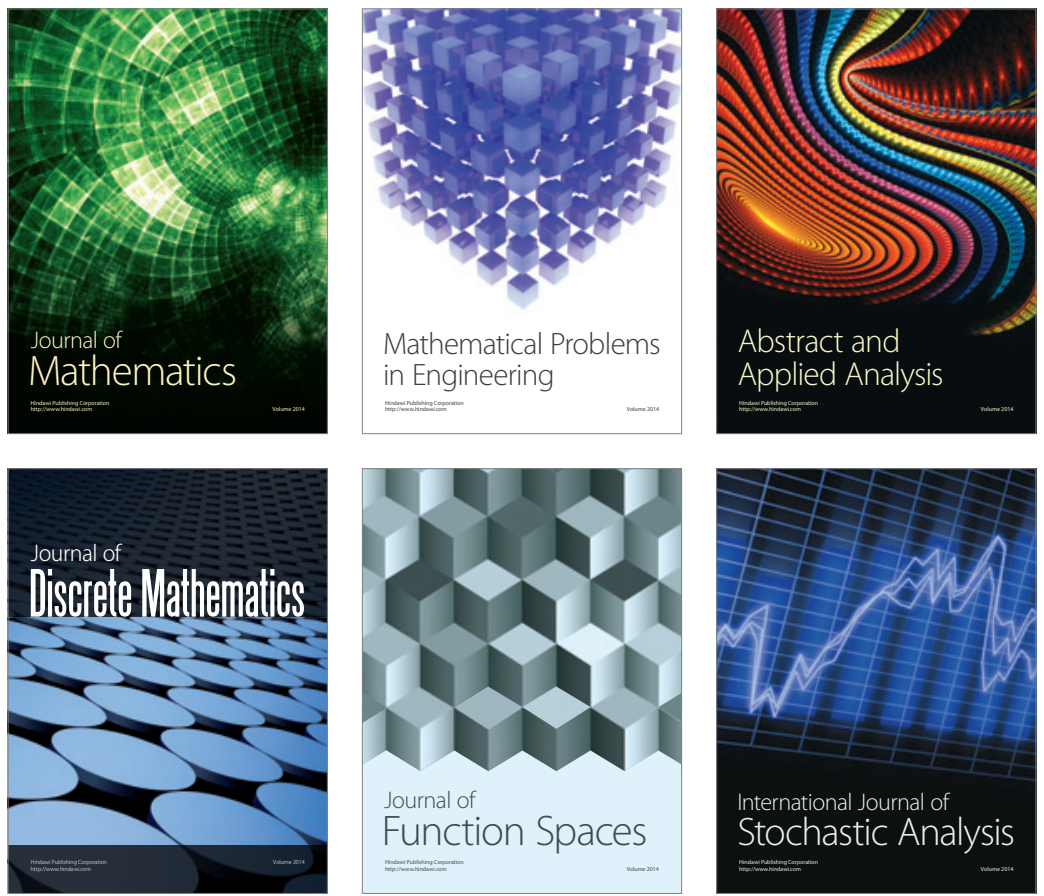

Journal of

Function Spaces



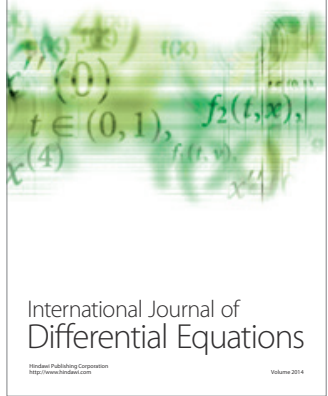
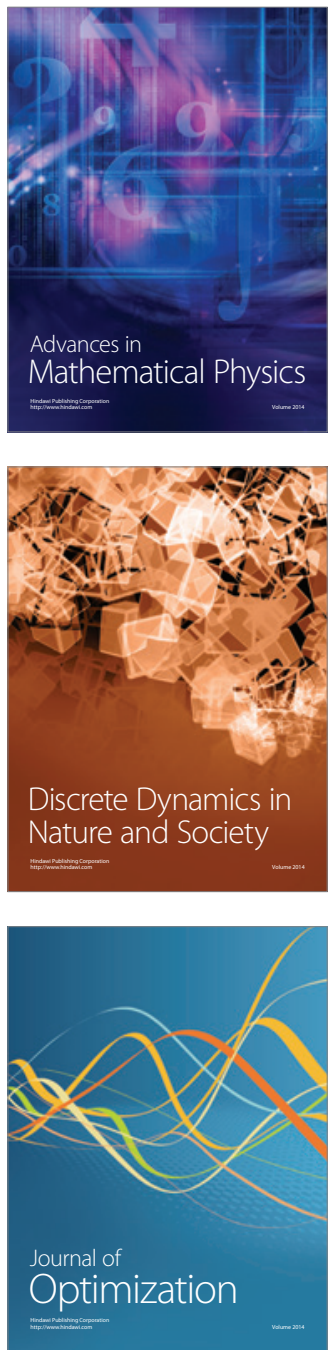